\section{"Processing Speed can Monitor Stimulant-Medication Effects in Adults with Attention Deficit Disorder with Hyperactivity": A Commentary}

Received: August 27, 2017; Accepted: August 29, 2017; Published: August 31, 2017

\author{
Elisabeth $\mathrm{H} \mathrm{Wiig}^{1 *}$ and \\ Niels P Nielsen ${ }^{2}$ \\ 1 Boston University and Knowledge \\ Research Institute, Inc., Arlington, Texas, \\ USA \\ 2 Västervik Sjukhus, Department of \\ Psychiatry, Västervik, Sweden
}

\section{*Corresponding author: Elisabeth H Wiig ehwiig@krii.com} of stimulant medication for adolescents and adults with attention deficit disorders with hyperactivity can be time consuming and may require repeated psychiatric consultations. In this article, we present a dose titration protocol that uses standardized processing-speed measures to achieve normalization of cognitive speed and set shifting costs, aspects of the symptomatology associated with ADHD, within a few hours [1,2].

In previous research with "A Quick Test of Cognitive Speed 2" we observed that a large majority (i.e., about 90\%) of adults with ADHD exhibited significantly slower cognitive speed and larger shift costs (overhead) during dual-dimension color-form naming than observed among neuro-typical adults [3-5]. These characteristics also differentiated adult psychiatric referrals with and without ADHD [6]. The findings suggested that a combination of reduced attention, working memory, set shifting and cognitive control, reflected in longer color-form naming times and larger shift costs (overhead) might characterize adults with ADHD. We then conducted a validation study in which we compared AQT processing speed measures in adults with ADHD without medication and with prescribed stimulant medication (methylphenidate) [7]. The results indicated that cognitive speed was significantly increased and shift costs (overhead) significantly reduced and generally normalized [3], when the same adults received their prescribed doses of immediate-release stimulant medication (methylphenidate). One of the limitations of the study was, however, that the prescribed variants and doses of the stimulant medication were not controlled. This led to the design and evaluation of a dose-titration protocol that introduced increasing, controlled doses of immediate release methylphenidate (Medikinet IR) associated with repeated measures of processing speed within a period of a few hours.

The dose-titration protocol used in this study measured processing speed for color, form and color-form naming at four points, each associated with a short psychiatric status interview. The assessments occurred, (1) with prior prescribed immediate-release stimulant medication, (2) after two days without medication, (3) with low-dose medication within 4555 min of ingesting 10/20 mg Medikinet IR, and (4) with high- dose medication within 45-55 min of ingesting an additional dose of $10 / 20 \mathrm{mg}$ Medikinet IR. Within the group of 40 patients we identified 7 non-responders for whom naming times for all measures changed minimally (i.e., within +/- 3 s) across treatments. There were 33 responders to medication for whom the changes in color-form naming times were larger than one standard deviation (i.e., $>6 \mathrm{~s}$ ) across treatments. Subsequent one-way ANOVA with post-hoc analyses, using lognormal transformation of naming times, indicated significant treatment effects for all processing-speed measures. Post hoc analyses (Scheffe) indicated significant increases in processing speed for single-dimension color and form naming with high dose, but not with low-dose medication, compared to the non-medication condition. For color-form naming, measures of demands on attention, working memory and cognitive control, processing speed increased significantly with low dose medication and further with high-dose medication, compared to the nomedication condition, but there was no statistical difference between the low- and high-dose conditions.

The results of the greatest clinical importance in this controlled dose-titration study were that cognitive speed increased incrementally with increased dosage. In the high-dose medication condition, both cognitive speed and set-shift costs (overhead) were reduced to normal levels (i.e., <3 s) [2-4], suggesting that the dosage might have been optimized. From a functional 
perspective, the cognitive changes observed should result in greater productivity [8], in work and daily-life settings and indirectly in a better quality of life. Whereas the dose-titration protocol was designed for use with immediate-release stimulant

\section{References}

1 Wiig EH, Nielsen NP, Minthon L, Warkentin S (2005) A quick test of cognitive speed. Et kort manual. Svensk versjon \& Norsk versjon. Harcourt/PsychCorp, Stockholm, Sweden.

2 Wiig EH, Nielsen NP, Minthon L (2017) A Quick Test of Cognitive Speed (AQT). Manual and app for ADHD. AQT Assessment ApS, Copenhagen, Denmark.

3 Wiig EH, Nielsen NP, Jacobson J (2007) A quick test of cognitive speed: group patterns of aging from 15 to 85 . Percept Mot Skills 104 1067-1075.

4 Nielsen NP, Wiig EH (2011) An additive model for relations between AQT single- and dual-dimension naming speed. Percept Mot Skills 112: 499-508. medication, it may be adapted for use with modified-release stimulant medications. We recognize that the dose-titration model and associated procedures should be validated in future research.

5 Nielsen NP, Wiig EH (2011) AQT cognitive speed differentiates adults with and without ADHD: A preliminary study. Int J Psychiatry Clin Pract 15: 187-193.

6 Wiig EH, Nielsen NP (2012) A quick test of cognitive speed for comparing processing speed and efficiency to differentiate adolescent and adult psychiatric referrals with and without attention-deficit/hyperactivity disorders. Prim Care Companion CNS Disord.

7 Nielsen NP, Wiig EH (2013) Validation of the AQT color-form additive model for screening and monitoring pharmacological treatment of ADHD. J Atten Dis 17: 187-193.

8 Advokat C (2010) What are cognitive effects of stimulant medication? Emphasis on adults with attention deficits/hyperactivity disorders (ADHD). Neurosci Bieobehav Rev 34: 1256-1266. 